\title{
CHARACTERIZATION OF UPPER SEMICONTINUOUSLY INTEGRABLE FUNCTIONS
}

\author{
ZOLTÁN BUCZOLICH
}

(Received 30 October 1992; revised 7 April 1993)

\begin{abstract}
We show that for a Henstock-Kurzweil integrable function $f$ for every $\epsilon>0$ one can choose an upper semicontinuous gage function $\delta$, used in the definition of the HK-integral if and only if $|f|$ is bounded by a Baire 1 function. This answers a question raised by $\mathrm{C}$. E. Weil.
\end{abstract}

1991 Mathematics subject classification (Amer. Math. Soc.): 26A39.

\section{Introduction}

It is known that if $f$ is a Henstock-Kurzweil integrable function then the gage function $\delta$, appearing in the definition of the HK-integral, can be chosen to be nearly upper semicontinuous, that is, $\delta$ equals an upper semicontinuous function almost everywhere. This result was obtained for $\mathbb{R}^{1}$ in Pfeffer [3] and for $\mathbb{R}^{m}$ in Buczolich [2]. C. E. Weil asked the author whether it is possible to find a characterization of those HK-integrable functions $f: \mathbb{R}^{m} \rightarrow \mathbb{R}$ for which the HK-integral can be defined by using upper semicontinuous gage functions.

In Pfeffer [3] it was shown that if a function is bounded and Lebesgue integrable then $\delta$ can be selected so that it is upper semicontinuous, that is, $f$ is upper semicontinuously integrable. Thus boundedness seems to play an important role in characterizations of upper semicontinuously integrable functions. Indeed, in our theorem we verify that $f$ is upper semicontinuously integrable if and only if there exists a Baire 1 function $g$ such that $g>|f|$. This property is also equivalent to the fact that any non-empty closed set has a portion on which $f$ is bounded. The proof of the one-dimensional case is somewhat simpler than the higher dimensional one. The higher dimensional version is related to some interesting combinatorial problems, namely to the chromatic number of maps consisting of certain non-overlapping interval systems in $\mathbb{R}^{m}$. The

Research supported by the Hungarian National Foundation for Scientific Research, Grant No. 2114.

(C) 1995 Australian Mathematical Society 0263-6115/95 \$A2.00+0.00 
details of this combinatorial question can be found in the statement of Lemma A.

\section{Preliminaries}

By $\mathbb{R}^{m}$ we denote $m$-dimensional Euclidean space. Given a set $A \subset \mathbb{R}^{m}$ we denote by $\operatorname{cl}(A)$, int $A, \partial A$ and $|A|$ the closure, the interior, the boundary and the Lebesgue measure of $A$. If $X \subset Y \subset \mathbb{R}^{m}$ then we denote the boundary and the interior of $X$ with respect to the subspace topology of $Y$ by $\partial_{Y} A$ and int $_{Y} A$ respectively. The open ball of radius $r$ centered at $x \in \mathbb{R}^{m}$ is denoted by $B(x, r)$. (In this paper we use the Euclidean metric; some papers use different but equivalent metrics in $\mathbb{R}^{m}$. The integral defined via any of these metrics is the same.) An $m$-dimensional interval is a set of the form $\left[a_{1}, b_{1}\right] \times \cdots \times\left[a_{m}, b_{m}\right]$. A collection $P=\left\{\left(A_{i}, x_{i}\right): i=1, \ldots, p\right\}$ is a subpartition of the interval $A$ if the intervals $A_{i} \subset A$ are non-overlapping and $x_{i} \in A_{i}$. The subpartition $P$ is a partition when $\bigcup_{i=1}^{P} A_{i}=A$. Given a positive function $\delta: A \rightarrow(0,+\infty)$ and a subpartition $P=\left\{\left(A_{i}, x_{i}\right): i=1, \ldots, p\right\}$ of $A$ we say that $P$ is $\delta$-fine when $A_{i} \subset B\left(x_{i}, \delta\left(x_{i}\right)\right)$.

If $f: A \rightarrow \mathbb{R}$ and $P$ is a subpartition of $A$ we put

$$
\sigma(f, P)=\sum_{i=1}^{p} f\left(x_{i}\right)\left|A_{i}\right|
$$

DEFINITION. Given an interval $A \subset \mathbb{R}^{m}$ a function $f: A \rightarrow \mathbb{R}$ is HK-integrable and its $\mathrm{HK}$-integral, $(\mathrm{HK}) \int_{A} f$, equals $I \in \mathbb{R}$ when for every $\epsilon>0$ there exists a function $\delta: A \rightarrow(0,+\infty)$ such that $|\sigma(f, P)-I|<\epsilon$ holds for any $\delta$-fine partition $P$ of $A$. In this paper we shall write $\int_{A} f$ instead of (HK) $\int_{A} f$.

If $f: A \rightarrow \mathbb{R}$ is HK-integrable and $\epsilon>0$ we denote by $\Delta(f, A, \epsilon)$ the set of those gage functions $\delta$ for which $|\sigma(f, P)-I|<\epsilon$ holds for any $\delta$-fine partition $P$ of $A$.

LEMMA (HENSTOCK). Assume that $A \subset \mathbb{B}^{m}$ is an interval, $f$ is HK-integrable on $A$, and $\epsilon>0$ is given. Then there is a gage function $\delta: A \rightarrow(0, \infty)$ such that

$$
\sum_{i=1}^{p}\left|f\left(x_{i}\right)\right| A_{i}\left|-\int_{A_{i}} f\right|<\epsilon
$$

for all $\delta$-fine subpartitions $P=\left\{\left(A_{i}, x_{i}\right): i=1, \ldots, p\right\}$ of $A$.

For the one-dimensional proofs see Pfeffer [4, Lemma 2.5], or [5, Lemma 3.12]. These one-dimensional proofs can easily be generalized also to the $m$-dimensional case.

Given $f: A \rightarrow \mathbb{R}$, an HK-integrable function, and $\epsilon>0$, we denote by $\Delta_{H}(f, A, \epsilon)$ the set of those gage functions $\delta$ for which (1) holds for all $\delta$-fine subpartitions of $A$. 
The proof of the Henstock Lemma shows that $\Delta(f, A, \epsilon / 3) \subset \Delta_{H}(f, A, \epsilon)$ (see for example Pfeffer [4]). Therefore the results of Pfeffer [3] and Buczolich [2] imply that if $f$ is HK-integrable on the interval $A$ then for any $\epsilon>0$ there exists a nearly upper semicontinuous $\delta \in \Delta_{H}(f, A, \epsilon)$. It follows from the Henstock Lemma that for $m=1$ the indefinite HK-integral $\int_{a}^{x} f$ is a continuous function see for example Pfeffer [5, Proposition 3.13]. It is not difficult to generalize the one-dimensional case to verify that if $f$ is HK-integrable on $A_{0}, A=\left[a_{1}, b_{1}\right] \times \cdots \times\left[a_{m}, b_{m}\right] \subset A_{0}$, and $A\left(h_{1}, \ldots, h_{2 m}\right)=\left[a_{1}+h_{1}, b_{1}+h_{2}\right] \times \cdots \times\left[a_{m}+h_{2 m-1}, a_{m}+h_{2 m}\right] \subset A_{0}$ then

$$
F\left(h_{1}, \ldots, h_{2 m}\right)=\int_{A\left(h_{1}, \ldots, h_{2 m}\right)} f
$$

is a continuous function of $2 m$ variables. We shall refer to this property as the continuity of the indefinite HK-integral. (In the literature a more general property used to be called the continuity of an interval function [5] but for this paper the above simple property is sufficient.)

\section{Main result}

This section is organized as follows. First we state our theorem which is followed by the statement of Lemma A. Then we prove the theorem by using Lemma A. Finally we present a proof of Lemma A.

THEOREM 1. Suppose that $f: A \rightarrow \mathbb{R}$ is HK-integrable. Then the following three statements are equivalent.

(i) For every $\epsilon>0$ there exists an upper semicontinuous $\delta \in \Delta(f, A, \epsilon)$.

(ii) For every non-empty closed subset $Q \subset A$ there exists an interval I such that $\operatorname{int}_{A}(I) \cap Q \neq \emptyset$ and $\left.f\right|_{\operatorname{int}_{A}(I) \cap Q}$ is bounded.

(iii) There exists a Baire 1 function $g: A \rightarrow \mathbb{R}$ such that $g(x)>|f(x)|$ for all $x \in A$.

Lemma A. Assume that $f$ is HK-integrable on $A \subset \mathbb{R}^{m}, \epsilon>0, \delta \in \Delta_{H}(f, A, \epsilon)$, $P=\left\{\left(A_{i}, x_{i}\right): i=1, \ldots, p\right\}$ is a subpartition of $A$, and for each $x_{i}$ there exists $a$ constant $K_{i}$ and a sequence $x_{i, n} \rightarrow x_{i}$ such that $B\left(x_{i, n}, \delta\left(x_{i, n}\right)\right) \supset A_{i}$ and $\left|f\left(x_{i, n}\right)\right|<$ $K_{i}$ for $n=1,2, \ldots$ and $i=1, \ldots, p$. Then there exists a constant $C \geq 1$ depending only on the dimension $m$ such that for each $i=1, \ldots, p$ there exists an $x_{i}^{\prime} \in$ $\left\{x_{i, 1}, x_{i, 2}, \ldots\right\}$ for which

$$
\sum_{i=1}^{p}\left|f\left(x_{i}^{\prime}\right)\right| A_{i}\left|-\int_{A_{i}} f\right|<C \epsilon .
$$


PROOF OF THE THEOREM. The proof consists of the steps: (i) implies (ii); (iii) implies (ii); (ii) implies (iii); and finally the dificult implication (ii) implies (i).

(i) implies (ii). In fact we verify that the negation of (ii) implies the negation of (i). Suppose that there exists a non-empty closed set $Q \subset A$ such that for every $K$ the set $\{x:|f(x)|>K\}$ is dense in $Q$. If $x_{0} \in Q$ is an isolated point of $Q$ then there exists an interval $I$ such that $Q \cap \operatorname{int}_{A}(I)=\left\{x_{0}\right\}$ and then $\left.f\right|_{\text {int }_{A}(I) \cap Q}$ is bounded by $\left|f\left(x_{0}\right)\right|$. Therefore $Q$ is perfect. Proceeding towards a contradiction, suppose that $\delta \in \Delta(f, A, 1)$ is upper semicontinuous. Since $\delta>0$ by Baire's theorem there exists $d>0$ and an interval $I_{0}$ such that $\operatorname{int}_{A}\left(I_{0}\right) \cap Q \neq \emptyset$ and $\{x \in Q: \delta(x)>d\}$ is dense in int $_{A}\left(I_{0}\right) \cap Q$. We can also assume that $d$ is less than the shortest side of $A$. Since $\delta$ is upper semicontinuous we have $\delta(x) \geq d$ for all $x \in \operatorname{int}_{A}\left(I_{0}\right) \cap Q$. Applying Baire's theorem again we can find $K_{1}>0$ and an interval $I_{1} \subset I_{0}$ such that $\operatorname{int}_{A}\left(I_{1}\right) \cap Q \neq \emptyset$ and $\left\{x:|f(x)|<K_{1}\right\}$ is dense in int $_{A}\left(I_{1}\right) \cap Q$. Let $K_{2}=2(2 \sqrt{m} / d)^{m}+K_{1}$. By our assumption $\{x:|f(x)|>K\}$ is dense in $Q$ for any $K$. Choose a point $x_{0} \in$ int $_{A}\left(I_{1}\right) \cap Q$ such that $\left|f\left(x_{0}\right)\right|>K_{2}$, and $\delta\left(x_{0}\right)>d$. We also choose a square $B \subset A$ such that $x_{0} \in \operatorname{int}_{A}(B), d / 2<\operatorname{diam}(B)<d$ (here we used the fact that $d$ is sufficiently small, that is, $d$ is less than the shortest side of $A$ and hence the square $B$ will fit into $A$ ). Denote by $P_{0}$ a fixed $\delta$-fine partition of $\operatorname{cl}(A \backslash B)$. Let $P_{1}=P_{0} \cup\left\{\left(B, x_{0}\right)\right\}$. It is obvious that $P_{1}$ is a $\delta$-fine partition of $A$. Since $\left\{x:|f(x)|<K_{1}\right\}$ is dense in $\operatorname{int}_{A}\left(I_{1}\right) \cap Q$ there exists an $x_{1} \in \operatorname{int}_{A}\left(I_{1}\right) \cap Q \subset \operatorname{int}_{A}\left(I_{0}\right) \cap Q$ such that $\left|f\left(x_{1}\right)\right|<K_{1}$ and $x_{1} \in B$. Then $P_{2}=P_{0} \cup\left\{\left(B, x_{1}\right)\right\}$ is also a $\delta$-fine partition of $A$. Thus

$\left|\sigma\left(f, P_{1}\right)-\sigma\left(f, P_{2}\right)\right|=\left|f\left(x_{0}\right)-f\left(x_{1}\right)\right| \cdot|B|>\left|2(2 \sqrt{m} / d)^{m}+K_{1}-K_{1}\right|(d / 2 \sqrt{m})^{m}=2$.

This contradicts the fact that $\delta$ was chosen for $\epsilon=1$.

(iii) implies (ii). If $Q$ has an isolated point then (ii) is valid. If $Q$ is non-empty and perfect then it is well-known that for any Baire 1 function, $g$, there exists a point $x_{0} \in Q$ such that $\left.g\right|_{Q}$ is continuous at $x_{0}$. Therefore one can easily find an interval $I$ such that $x_{0} \in \operatorname{int}_{A}(I)$ and $\left.g\right|_{\operatorname{int}_{A}(I) \cap Q}$ is bounded. Then $\left.f\right|_{\text {int }_{A}(I) \cap Q}$ is also bounded.

(ii) implies (iii). Put $Q_{0}=A$. Assume that $r$ is not a limit ordinal and $Q_{r-1} \neq \emptyset$ is a given closed set. By using (ii) choose an interval $I_{r-1}$ such that int ${ }_{A}\left(I_{r-1}\right) \cap Q_{r-1} \neq \emptyset$ and $\left.f\right|_{\text {int }_{A}\left(I_{r-1}\right) \cap Q_{r-1}}$ is bounded. Put $Q_{r}=Q_{r-1} \backslash \operatorname{int}_{A}\left(I_{r-1}\right)$. Since $A$ is closed in $\mathbb{R}^{m}$ the set $Q_{r}$ is also closed in $\mathbb{R}^{m}$, not only in the subspace topology of $A$. If $r$ is a limit ordinal put $Q_{r}=\bigcap_{s<r} Q_{s}$. It is well-known that any well-ordered decreasing sequence of closed sets in $\mathbb{R}^{m}$ is of cardinality less than $\aleph_{1}$. It is also easy to see that the sets int ${ }_{A}\left(I_{r}\right) \cap Q_{r}$ are disjoint and $\bigcup_{r}\left(\right.$ int $\left._{A}\left(I_{r}\right) \cap Q_{r}\right)=A$. Therefore the sets of the form int ${ }_{A}\left(I_{r}\right) \cap Q_{r}$ can be ordered into a sequence denoted by $H_{1}, H_{2}, \ldots, H_{n}, \ldots$ so that they are pairwise disjoint and $\bigcup_{n=1}^{\infty} H_{n}=A$. It is also clear that $f$ is bounded on the sets $H_{n}$, and the sets $H_{n}$ are of type $F_{\sigma}$. Choose integer constants $K_{n}$ such that $K_{1}<K_{2}<\cdots<K_{n}<\cdots$ and $|f|$ is bounded on $H_{n}$ by $K_{n}$. Assume that $H_{n}=\bigcup_{i=1}^{\infty} F_{n, i}$, where the sets $F_{n, i}$ are closed, and $F_{n, 1} \subset F_{n, 2} \subset \ldots$. Define the 
function $g_{j}$ so that $g_{j}(x)=K_{n}$ if $x \in F_{n, j}$ and $n \leq j$. Since for a fixed $j$ the closed sets $F_{n, j} \subset H_{n}$ are disjoint for $n=1,2, \ldots$ the function $g_{j}(x)$ is continuous on $\bigcup_{n=1}^{j} F_{n, j}$. Since $\mathbb{R}^{m}$ is a normal space by [1, Satz VIII] one can extend $g_{j}$ to be defined and continuous on $\mathbb{R}^{m}$. If $x \in A$ then there exists exactly one $n(x)$ such that $x \in H_{n(x)}$ and for $j>j(x)$ we also have $x \in F_{n(x), j}$. Therefore $\lim _{j \rightarrow \infty} g_{j}(x)=K_{n}$. Thus the function defined by $g=\lim _{j \rightarrow \infty} g_{j}$ is Baire 1 and $g(x)=K_{n(x)}>|f(x)|$ if $x \in H_{n(x)}$.

(ii) implies (i). Assume that $\epsilon>0$ is given and (ii) holds. Recall that $\Delta_{H}\left(f, A, \epsilon_{0}\right)$ contains a nearly upper semicontinuous, and hence measurable gage function for any $\epsilon_{0}>0$. Choose a measurable $\delta_{H} \in \Delta_{H}(f, A, \epsilon / 4 C)$, where $C$ is the constant given in Lemma A. Put $W_{0}=A$. If $W_{s}$ is defined for ordinals $s<r<\aleph_{1}$ and $r$ is a limit ordinal put $W_{r}=\cap_{s<r} W_{s}$.

If $r=s+1$ and $W_{s}$ contains an isolated point $w_{s}$ put $E_{s}=\left\{w_{s}\right\}=T_{s}, \eta_{s}=$ $\delta_{H}\left(w_{s}\right) / 2$ and $W_{r}=W_{s} \backslash E_{s}$. Also choose an interval $I_{s}$ such that int $t_{A}\left(I_{s}\right) \cap W_{s}=E_{s}$ and diam $I_{s}<\eta_{s}$.

If $r=s+1$ and $W_{s}$ is non-empty and perfect then by using (ii) and Baire's theorem we can choose an interval $I_{s}$ such that $E_{s}=\operatorname{int}_{A}\left(I_{s}\right) \cap W_{s} \neq \emptyset,|f|$ is bounded by $K_{s}$ on $E_{s}$, and there exists an $\eta_{s}>0$ such that

$$
T_{s}=\left\{x \in E_{s}: \delta_{H}(x)>\eta_{s}\right\}
$$

is dense in $E_{s}$. We can also assume that $\operatorname{diam} I_{s}<\eta_{s}$. Put $W_{r}=W_{s} \backslash E_{s}$.

Since a well-ordered strictly decreasing sequence of closed sets of $\mathbb{R}^{m}$ terminates at an ordinal number $\alpha<\aleph_{1}$ we can assume that $W_{\alpha} \neq \emptyset$ and $W_{\alpha+1}=\emptyset$. We remark that the sets $W_{s} \subset A$ are bounded and closed. Hence if $W_{s} \neq \emptyset$ for $s<r$ then $\bigcap_{s<r} W_{s} \neq \emptyset$ and therefore the sequence $W_{s}$ cannot terminate at a limit ordinal. Clearly $E_{\alpha}=W_{\alpha}$. We shall denote by $\Lambda$ the set of those ordinals which are less than $\alpha+1$. It is clear that $\Lambda$ is countable and hence we can find an injective function $j: \Lambda \rightarrow \mathbb{N}$.

If $s \in \Lambda$ and $\left|E_{s}\right|=0$ then put $\kappa_{s}=\eta_{s}$, and choose an open set $U_{s} \supset E_{s}$ such that

$$
\left|U_{s}\right|<\epsilon /\left(2 \cdot 2^{j(s)} K_{s}\right),
$$

and $\partial I_{s} \subset U_{s}$.

If $s \in \Lambda$ and $\left|E_{s}\right|>0$ then using the fact that $\delta_{H}$ is measurable and positive we can find $0<\kappa_{s} \leq \eta_{s}$ such that letting $V_{s}=\left\{x \in E_{s}: \delta_{H}(x) \leq \kappa_{s}\right\}$ we have $\left|V_{s}\right|<\epsilon /\left(16 \cdot 2^{j(s)} K_{s}\right)$. The measurability of $\delta_{H}$ also implies the existence of a non-empty open set $U_{s} \supset V_{s}$ such that

$$
\left|U_{s}\right|<\epsilon /\left(16 \cdot 2^{j(s)} K_{s}\right),
$$

and we can also assume that $U_{s}$ contains $\partial I_{s}$. 
If $s \in \Lambda$ and $x \in U_{s} \cap E_{s}$ put

$$
\delta(x)=\min \left\{\operatorname{dist}\left(x, \partial_{A} I_{s}\right), \operatorname{dist}\left(x, \mathbb{R}^{m} \backslash U_{s}\right), 1 / j(s)\right\} .
$$

It is obvious that

$$
B(x, \delta(x)) \cap A \subset U_{s} \cap I_{s} \text { for } \quad x \in U_{s} \cap E_{s} .
$$

If $x \in E_{s} \backslash U_{s}$ put

$$
\delta(x)=\min \left\{\operatorname{dist}\left(x, \partial_{A} I_{s}\right), 1 / j(s), \kappa_{s}\right\} .
$$

Now $\delta$ is defined for all $s \in \Lambda$ and it is clear that $\delta$ is positive. Observe that if $y \in \partial_{A} I_{s}$ then

$$
\delta(x) \leq \operatorname{dist}\left(x, \partial_{A} I_{s}\right) \leq \operatorname{dist}(x, y) .
$$

Since $W_{s}$ is closed and $U_{s}$ contains the boundary of $I_{s}$ the set $E_{s} \backslash U_{s}$ is closed. It is obvious that $\delta$ is upper semicontinuous on the set $E_{s} \backslash U_{s}$. It is also clear from its definition that $\delta$ is upper semicontinuous on the set $E_{s} \cap U_{s}$, and if $x \notin E_{s} \cap U_{s}$, $x_{n} \rightarrow x, x_{n} \in E_{s} \cap U_{s}(n=1,2, \ldots)$ then $\delta\left(x_{n}\right) \rightarrow 0$.

$(\alpha)$ The above properties imply that $\delta$ is upper semicontinuous on $E_{s}$.

$(\beta)$ Assume that $x_{n} \rightarrow y$, and for any $s \in \Lambda$ we have $x_{n} \in E_{s}$ for only finitely many indices $n$. For any $\nu>0$ there exists only finitely many indices $s \in \Lambda$ for which $1 / j(s) \geq v$. Therefore from $\delta(x) \leq 1 / j(s)$ when $x \in E_{s}$, it follows that $\delta\left(x_{n}\right) \geq v$ can hold only for finitely many $n$ 's and hence $\delta\left(x_{n}\right) \rightarrow 0$ as $n \rightarrow \infty$. Thus $\lim _{n \rightarrow \infty} \delta\left(x_{n}\right)=0<\delta(y)$.

$(\gamma)$ If $x_{n} \in E_{s}=\operatorname{int}_{A}\left(I_{s}\right) \cap W_{s}$ for $n=1,2, \ldots$, and $x_{n} \rightarrow y \notin E_{s}$ then using that $W_{s}$ is closed we obtain $y \in \partial_{A} I_{s}$ and (6) implies that $\delta\left(x_{n}\right) \leq \operatorname{dist}\left(x_{n}, y\right)$.

If $y \in A$ then there exists exactly one $r \in \Lambda$ for which $y \in E_{r}$. Assume that $x_{n} \rightarrow y$. For any natural number $n$ there exists exactly one $s(n) \in \Lambda$ for which $x_{n} \in E_{s(n)}$. For $s \in \Lambda$ put $N_{s}=\left\{n: x_{n} \in E_{s}\right\}$. Put $M_{1}=N_{r}, M_{2}=\left\{n: s(n) \neq r\right.$ and $N_{s(n)}$ is finite $\}$, and $M_{3}=\mathbb{N} \backslash\left(M_{1} \cap M_{2}\right)=\left\{n: s(n) \neq r\right.$ and $N_{s(n)}$ is infinite $\}$. If $M_{2}$ is infinite then $(\beta)$ is applicable to the subsequence $\left\{x_{n}: n \in M_{2}\right\}$. If $n^{\prime} \in M_{3}$ then $(\gamma)$ is applicable to the subsequence $\left\{x_{n}: n \in N_{s\left(n^{\prime}\right)}\right\}$ and we obtain $\delta\left(x_{n^{\prime}}\right) \leq \operatorname{dist}\left(x_{n^{\prime}}, y\right)$. This implies $\lim _{n \rightarrow \infty, n \in M_{3}} \delta\left(x_{n}\right)=0$. Thus by splitting $\left\{x_{n}\right\}$ into at most three subsequences and using $(\alpha),(\beta)$, and $(\gamma)$ we obtain that $\lim _{\sup _{n \rightarrow \infty}} \delta\left(x_{n}\right) \leq \delta(y)$ proving that $\delta$ is upper semicontinuous on $A$.

Next we have to verify that $\delta \in \Delta(f, A, \epsilon)$. For an $x \in A$ denote by $r(x)$ the index in $\Lambda$ for which $x \in E_{r(x)}$.

Assume that $\left\{\left(A_{i}, x_{i}\right): i=1, \ldots, p\right\}$ is a $\delta$-fine partition of $A$. Then

$$
\sum_{i=1}^{p}\left|f\left(x_{i}\right)\right| A_{i}\left|-\int_{A_{i}} f\right|=\sum_{s \in \Lambda} \sum_{\left\{i: x_{i} \in E_{s}\right\}}\left|f\left(x_{i}\right)\right| A_{i}\left|-\int_{A_{i}} f\right|=\Psi_{1}
$$


where the 'transfinite' sum $\sum_{s \in \Lambda}$ can be defined since there are only finitely many indices $s$ for which there exists $x_{i}$ such that $r\left(x_{i}\right)=s$. By definition, the empty sum and the transfinite sum of zeros equal zero.

Put

$$
\Psi_{2}=\sum_{s \in \Lambda} \sum_{\left\{i: x_{i} \in E_{s} \cap U_{s}\right\}}\left|f\left(x_{i}\right)\right| A_{i}\left|-\int_{A_{i}} f\right|,
$$

and

$$
\Psi_{3}=\sum_{s \in \Lambda} \sum_{\left\{i: x_{i} \in E_{s} \backslash U_{s}\right\}}\left|f\left(x_{i}\right)\right| A_{i}\left|-\int_{A_{i}} f\right| .
$$

Obviously $\Psi_{1}=\Psi_{2}+\Psi_{3}$. If $x_{i} \in E_{s} \backslash U_{s}$ then $\left|E_{s}\right|>0$ and $x_{i} \notin V_{s}$; therefore $\delta_{H}\left(x_{i}\right)>$ $\kappa_{s} \geq \delta\left(x_{i}\right)$. Hence the partition in $A$ defined by $\left\{\left(A_{i}, x_{i}\right): \exists s \in \Lambda\right.$ such that $x_{i} \in$ $\left.E_{s} \backslash U_{s}\right\}$ is $\delta_{H}$-fine. Thus $\Psi_{3}<\epsilon / 4 C<\epsilon / 2$ where we used the fact that $\delta_{H} \in$ $\Delta_{H}(f, A, \epsilon / 4 C)$ and $C \geq 1$.

To estimate $\Psi_{2}$ assume in this paragraph that an $s \in \Lambda$ is given. The set $T_{s}$, introduced during the definition of $E_{s}$, is dense in $E_{s}$. By (5) for any $x \in E_{s} \cap U_{s}$, we have $B(x, \delta(x)) \cap A \subset I_{s}$. Since diam $I_{s}<\eta_{s}$ by using (2) we have $B\left(x, \delta_{H}(x)\right) \supset$ $I_{s} \supset B(y, \delta(y)) \cap A$ for any $x \in T_{s}, y \in E_{s} \cap U_{s}$. Thus for any $x_{i} \in E_{s} \cap U_{s}$ there exists a sequence $x_{i, n} \rightarrow x_{i}$ such that $x_{i, n} \in T_{s}$ and hence $B\left(x_{i, n}, \delta_{H}\left(x_{i, n}\right)\right) \supset I_{s} \supset$ $B\left(x_{i}, \delta\left(x_{i}\right)\right) \cap A \supset A_{i}$, for $n=1,2, \ldots$ and $\left|f\left(x_{i, n}\right)\right|<K_{s}$.

The above argument is valid for any $s \in \Lambda$. Observe that Lemma $\mathrm{A}$ is applicable with $f=f, A=A, \epsilon=\epsilon / 4 C, \delta=\delta_{H}, P=\left\{\left(A_{i}, x_{i}\right): i \in\{1, \ldots, p\}, \exists s \in \Lambda, x_{i} \in\right.$ $\left.E_{s} \cap U_{s}\right\}$, and for $x_{i} \in E_{s} \cap U_{s}$ choose $K_{i}=K_{s}$ and $x_{i, n}=x_{i, n}(n=1,2, \ldots)$. We obtain that for any $i$ for which $x_{i} \in E_{s} \cap U_{s}$ there exists an $x_{i}^{\prime} \in T_{s}$ such that

$$
\Psi_{4}=\sum_{s \in \Lambda} \sum_{\left\{i: x_{i} \in E_{s} \cap U_{s}\right\}}\left|f\left(x_{i}^{\prime}\right)\right| A_{i}\left|-\int_{A_{i}} f\right|<\epsilon / 4 .
$$

Recall that $|f|$ is bounded by $K_{s}$ on $E_{s}$. For $x_{i} \in E_{s} \cap U_{s}$, by (5) we have $A_{i} \subset B\left(x_{i}, \delta\left(x_{i}\right)\right) \cap A \subset U_{s}$. Therefore using (4) we obtain

$$
\sum_{\left\{i: x_{i} \in E_{s} \cap U_{s}\right\}}\left|f\left(x_{i}\right)\right|\left|A_{i}\right|<K_{s} \cdot \sum_{\left\{i: x_{i} \in E_{s} \cap U_{s}\right\}}\left|A_{i}\right|<K_{s}\left|U_{s}\right|<K_{s} \frac{\epsilon}{16 \cdot 2^{j(s)} K_{s}}=\frac{\epsilon}{16 \cdot 2^{j(s)}} .
$$

Thus we have

$$
\Psi_{5}=\sum_{s \in \Lambda} \sum_{\left\{i: x_{i} \in E_{s} \cap U_{s}\right\}}\left|f\left(x_{i}\right)\right| \cdot\left|A_{i}\right|<\sum_{j=1}^{\infty} \frac{\epsilon}{16 \cdot 2^{j}}=\epsilon / 16 .
$$

Since $x_{i}^{\prime} \in T_{s} \subset E_{s}$ implies $\left|f\left(x_{i}^{\prime}\right)\right|<K_{s}$ one can obtain similarly to the previous estimation

$$
\Psi_{6}=\sum_{s \in \Lambda} \sum_{\left\{i: x_{i} \in E_{s} \cap U_{s}\right\}}\left|f\left(x_{i}^{\prime}\right)\right| \cdot\left|A_{i}\right|<\epsilon / 16 .
$$


From (7) and (9) we obtain that

$$
\Psi_{7}=\sum_{s \in \Lambda} \sum_{\left\{i: x_{i} \in E_{s} \cap U_{s}\right\}}\left|\int_{A_{i}} f\right|<\frac{\epsilon}{4}+\Psi_{6}<\frac{\epsilon}{4}+\frac{\epsilon}{16} .
$$

Finally using (8) and (10) we infer

$$
\Psi_{2}<\Psi_{5}+\Psi_{7}<\frac{\epsilon}{16}+\frac{\epsilon}{4}+\frac{\epsilon}{16}<\frac{\epsilon}{2} .
$$

Therefore $\Psi_{1}=\Psi_{2}+\Psi_{3}<\epsilon / 2+\epsilon / 2=\epsilon$. This proves $\delta \epsilon \Delta_{H}(f, A, \epsilon) \subset$ $\Delta(f, A, \epsilon)$ and concludes the proof of the theorem.

PROOF OF LEMMA A. First we prove the one-dimensional case. If we order the intervals $A_{i}$ according to the ordering of the real line, then taking every second interval $A_{i}$ the set $\{1, \ldots, p\}$ can be split into two subsets, $N_{1}$ and $N_{2}$ such that $N_{1} \cup N_{2}=\{1, \ldots, p\}, N_{1} \cap N_{2}=\emptyset$ and the (closed) intervals $\left\{A_{i}: i \in N_{j}\right\}$ are pairwise disjoint for $j=1,2$.

If $x_{i} \in \operatorname{int}_{A}\left(A_{i}\right)$ then using the assumption of Lemma $\mathrm{A}$ one can choose an $x_{i, n} \in$ int $_{A}\left(A_{i}\right)$. Put $x_{i}^{\prime}=x_{i, n}$, and $A_{i}^{\prime}=A_{i}$.

If $x_{i}$ is one of the endpoints of $A_{i}$ then using the fact that the indefinite HK-integral is a continuous function by enlarging slightly the intervals $A_{i}$ one can obtain the intervals $A_{i}^{\prime}$ such that

(i) $x_{i} \in \operatorname{int}_{A}\left(A_{i}^{\prime}\right)$ and hence there exists an $n(i)$ such that $x_{i}^{\prime}=x_{i, n(i)}$ belongs to

(ii) $A_{i}^{\prime}$ and $A_{i}^{\prime} \subset B\left(x_{i}^{\prime}, \delta\left(x_{i}^{\prime}\right)\right)$,

$$
\left|\int_{A_{i}} f-\int_{A_{i}^{\prime}} f\right|<\frac{\epsilon}{2 p}
$$

(iii)

$$
K_{i}|| A_{i}^{\prime}|-| A_{i}||<\frac{\epsilon}{2 p}
$$

(iv) the intervals $\left\{A_{i}^{\prime}: i \in N_{j}\right\}$ are non-overlapping for $j=1,2$.

By (i) we have $A_{i}^{\prime} \subset B\left(x_{i}^{\prime}, \delta\left(x_{i}^{\prime}\right)\right)$. This and (iv) implies that the subpartitions $\left\{\left(A_{i}^{\prime}, x_{i}^{\prime}\right): i \in N_{j}\right\}$ are $\delta$-fine in $A$ for $j=1,2$. Thus

$$
\sum_{i \in N_{j}}\left|f\left(x_{i}^{\prime}\right)\right| A_{i}^{\prime}\left|-\int_{A_{i}^{\prime}} f\right|<\epsilon
$$


holds for $j=1,2$. Therefore

$$
\sum_{i=1}^{p}\left|f\left(x_{i}^{\prime}\right)\right| A_{i}^{\prime}\left|-\int_{A_{i}^{\prime}} f\right|<2 \epsilon .
$$

Finally

$$
\begin{aligned}
\sum_{i=1}^{p} & \left|f\left(x_{i}^{\prime}\right)\right| A_{i}\left|-\int_{A_{i}} f\right| \\
& \leq \sum_{i=1}^{p}\left|f\left(x_{i}^{\prime}\right)\right| A_{i}\left|-\int_{A_{i}} f-f\left(x_{i}^{\prime}\right)\right| A_{i}^{\prime}\left|+\int_{A_{i}^{\prime}} f\right|+\sum_{i=1}^{p}\left|f\left(x_{i}^{\prime}\right)\right| A_{i}^{\prime}\left|-\int_{A_{i}^{\prime}} f\right| \\
& \leq \sum_{i=1}^{p}\left|f\left(x_{i}^{\prime}\right)\right| \cdot|| A_{i}^{\prime}|-| A_{i}||+\sum_{i=1}^{p}\left|\int_{A_{i}} f-\int_{A_{i}^{\prime}} f\right|+2 \epsilon<3 \epsilon
\end{aligned}
$$

where at the last inequality we used (ii) and (iii). Thus when $m=1$ we can choose $C=3$ and this concludes the proof of the one-dimensional case.

To generalize the proof of the one-dimensional case the following statement would be useful.

STATEMENT. There exists a constant $K$ depending only on the dimension such that given any finite collection $\left\{A_{i}: i=1, \ldots, p\right\}$ of non-overlapping intervals in $\mathbb{R}^{m}$, they can be colored by $K$ colors, that is, there exists a function $h:\{1, \ldots, p\} \rightarrow$ $\{1, \ldots, K\}$, such that intervals of the same color, that is, $\left\{A_{i}: h(i)=j\right\}$, are disjoint for all $j \in\{1, \ldots, K\}$.

For $m=1,2$ the statement is true, although the best possible value of $K$ for $m=2$ seems to be unknown. When $m \geq 3$ then it is not known whether the statement is true or not.

If $\rho \geq 1$ is fixed and we are looking at $\rho$-regular coverings, that is, the ratio of the longest and shortest side of each $A_{i}$ is bounded by $\rho$, then the statement is true. As B. Kirchheim pointed out to the author the ideas used in the proof of Ziemer $[6,1.3 .5$. Theorem, p. 9-12] can be generalized to this case.

Fortunately to prove Lemma $\mathrm{A}$ it is not necessary to prove the statement. We shall show that we can shrink the intervals $A_{i}$ slightly to obtain the intervals $A_{i}^{\prime \prime}$ for which the statement and Lemma A holds. Using the continuity of the HK-integral for $i=1, \ldots p$ choose $0<\gamma_{i}<1$ such that if we denote by $A_{i}^{\prime \prime}$ the image of $A_{i}$ under the affine transformation $T_{i}(x)=x_{i}+\gamma_{i}\left(x-x_{i}\right)$ then

$$
K_{i}|| A_{i}|-| A_{i}^{\prime \prime}||<\epsilon / 2 p, \quad \text { and } \quad\left|\int_{A_{i}} f-\int_{A_{i}^{\prime \prime}} f\right|<\epsilon / 2 p
$$


holds for $i=1, \ldots, p$. It is clear that if there exists $C_{0}$ depending only on $m$ and $x_{i}^{\prime} \in\left\{x_{i, 1}, x_{i, 2}, \ldots\right\}$ such that

$$
\sum_{i=1}^{p}\left|f\left(x_{i}^{\prime}\right)\right| A_{i}^{\prime \prime}\left|-\int_{A_{i}^{\prime \prime}} f\right|<C_{0} \epsilon
$$

then

$$
\sum_{i=1}^{p}\left|f\left(x_{i}^{\prime}\right)\right| A_{i}\left|-\int_{A_{i}} f\right|<C_{0} \epsilon+\sum_{i=1}^{p} K_{i} \cdot|| A_{i}|-| A_{i}^{\prime \prime}||+\sum_{i=1}^{p}\left|\int_{A_{i}} f-\int_{A_{i}^{\prime \prime}} f\right|<\left(C_{0}+1\right) \epsilon
$$

and Lemma A holds with $C=C_{0}+1$.

For ease of notation we work out the two-dimensional case; the higher dimensional ones are similar. Divide the index set $\{1, \ldots, p\}$ into 9 disjoint subsets $N_{1}, N_{2}, \ldots, N_{9}$. If $x_{i} \in \operatorname{int}_{A}\left(A_{i}\right)$ then let $i \in N_{1}$. If $x_{i}$ is not a vertex of $A_{i}$ and $x_{i}$ is on the lower side of $A_{i}$ then let $i$ be in $N_{2}$. The index sets $N_{3}, N_{4}$, and $N_{5}$ are defined like $N_{2}$ by using the right, upper, and left sides of $A_{i}$ in the definition respectively. If $x_{i}$ is the lower left (lower right, upper right, upper left) vertex of $A_{i}$ then $i$ is in $N_{6},\left(N_{7}, N_{8}, N_{9}\right)$ respectively.

Since the intervals $A_{i}$ are non-overlapping it is obvious that $\left\{\left(A_{i}^{\prime \prime}, x_{i}\right): i \in N_{1}\right\}$ consists of disjoint intervals, and a moment's reflection shows that each of the subsubpartitions $\left\{\left(A_{i}^{\prime \prime}, x_{i}\right): i \in N_{j}\right\}$ also consists of disjoint intervals when $j=2, \ldots, 9$. Thus with $K=9$ the conclusion of the statement holds for the intervals $\left\{A_{i}^{\prime \prime}: i=\right.$ $1, \ldots, p\}$.

Assume now that $j \in\{1,2, \ldots, 9\}$ is fixed. Since the intervals $\left\{A_{i}^{\prime \prime}: i \in N_{j}\right\}$ are disjoint one can enlarge the intervals $A_{i}^{\prime \prime}$ slightly and obtain the intervals $A_{i}^{\prime}$ such that $x_{i} \in \operatorname{int}\left(A_{i}^{\prime}\right)$ and properties (i)-(iv) at the beginning of the proof of the one-dimensional case of Lemma A hold with $A_{i}^{\prime \prime}$ instead of $A_{i}$ and for $j=1, \ldots, 9$. Replacing $A_{i}$ by $A_{i}^{\prime \prime}$ in the rest of the computations used for the one-dimensional case one can obtain that

$$
\sum_{i=1}^{p}\left|f\left(x_{i}^{\prime}\right)\right| A_{i}^{\prime \prime}\left|-\int_{A_{i}^{\prime \prime}} f\right|<10 \epsilon
$$

and hence (11) holds with $C_{0}=10$. This concludes the proof for $m=2$. When $m \geq 3$ then a similar separation of cases according to the location of $x_{i}$ on the boundary of $A_{i}$ can be used. This concludes the proof of Lemma A.

\section{References}

[1] P. Alexandroff and H. Hopf, Topologie (Chelsea, New York, 1965)

[2] Z. Buczolich, 'Nearly upper semicontinuous gage functions in $\mathbb{R}^{m}$ ', Real Anal. Exchange 13 (1987-88), 245-252. 
[3] W. F. Pfeffer, 'A note on the generalized Riemann integral', Proc. Amer. Math. Soc. 103 (1988), 1161-1166.

[4] - 'A Riemann type integration and the fundamental theorem of calculus', Rend. Circolo Mat. Palermo (2), XXXVI (1987), 482-506.

[5] _ 'Lectures on geometric integration and the divergence theorem', Rend. Istit. Mat. Univ. Trieste, in press.

[6] W. P. Ziemer, Weakly differentiable functions (Springer, Berlin, 1989).

Department of Analysis

Eötövos Loránd University

Budapest

Muzeum krt 6-8, Hungary, H- 1088

e-mail: buczo@konig.elte.hu 